\title{
(6) OPEN ACCESS \\ Percutaneous coronary intervention in the UK: recommendations for good practice 2015
}

\author{
Adrian P Banning, ${ }^{1}$ Andreas Baumbach, ${ }^{2}$ Dan Blackman, ${ }^{3}$ Nick Curzen, ${ }^{4}$ \\ Sen Devadathan, ${ }^{5}$ Douglas Fraser, ${ }_{1}^{6}$ Peter Ludman, ${ }^{7}$ Micheal Norell, ${ }^{8}$ Dougie Muir, ${ }^{9}$ \\ James Nolan, ${ }^{10}$ Simon Redwood, ${ }^{11}$ On behalf of the British Cardiovascular \\ Intervention society
}

- Additional material is published online only. To view please visit the journal online (http://dx.doi.org/10.1136/ heartjnl-2015-307821)

For numbered affiliations see end of article.

\section{Correspondence to} Professor Adrian Banning, Oxford Heart Centre, Oxford University Hospitals, Headley Way, Oxford OX39DU, UK; adrian.banning@ouh.nhs.uk

Received 16 March 2015 Revised 14 April 2015 Accepted 15 April 2015

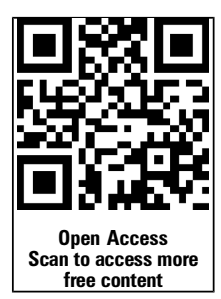

CrossMark

To cite: Banning AP, Baumbach A, Blackman D, et al. Heart 2015;101:1-13.

\section{ABSTRACT}

Over the last 35 years, there has been dramatic progress in the technology and applicability of percutaneous techniques to treat obstructive coronary heart disease. Percutaneous coronary intervention (PCI) has a considerable evidence base and it is firmly established as the most common procedure used in the invasive treatment of patients with coronary heart disease in the UK. This set of guidelines aims to address specifically issues relating to $\mathrm{PCl}$ and not the growing subspecialty of structural heart disease intervention. It is not intended to provide a review of the entire evidence base for coronary intervention. The evidence base relating to $\mathrm{PCl}$ is extensively reviewed in international guidelines and the British Cardiovascular Intervention society endorses these guidelines and their updates. The guidelines presented here focus on issues pertinent to practice within the UK and set out a recommended template to ensure optimal delivery of patient care.

\section{INTRODUCTION}

Over the last 35 years, there has been dramatic progress in the technology and applicability of percutaneous techniques to treat obstructive coronary heart disease (CHD). Percutaneous coronary intervention (PCI) has a considerable evidence base and it is firmly established as the most common procedure used in the invasive treatment of patients with $\mathrm{CHD}$ in the UK.

In a field where technology continues to develop rapidly, it is essential that centres undertaking PCI are appropriately equipped, for staff to be competent and for case selection to be matched to the skill of the operators. This is the fourth set of guidelines produced by the British Cardiovascular Intervention Society (BCIS) and the British Cardiovascular Society (BCS) and it replaces the Guideline published in 2005. Considerable developments have occurred during the last 10 years including the widespread application of emergency primary PCI (PPCI) for treatment of patients with ST segment elevation myocardial infarction (STEMI). Providing comprehensive data remains an essential requirement both for the centres and operators performing PCI. The BCIS audit of adult interventional procedures, run in collaboration with the National Institute for Cardiovascular Outcomes Research (NICOR) allows each patient's outcome to be tracked through his or her individual cardiac journey from birth until death, in turn enabling the UK's national data to be analysed, presented and reviewed. Within the last year individual PCI operator outcomes of PCI procedures have been published in the public domain.

There has been a slowing in the previously exponential increase in rate of PCI in the UK within the last few years. The need for emergency cardiac surgery following failed angioplasty remains, but this is now very infrequent. A large proportion of PCI is undertaken without on-site surgery and the linkage of these sites within Cardiac Networks remains fundamental to the delivery of optimal patient care, particularly when considering surgical revascularisation options. A common standard is applied in assessing a site's suitability for the performance of PCI and this is independent of the provision of on-site surgery. Sites performing emergency PPCI have specific requirements which exceed those of sites performing only selected elective and urgent cases.

This set of guidelines aims to address specifically issues relating to PCI and not the growing subspecialty of structural heart disease intervention. It is not intended to provide a review of the entire evidence base for coronary intervention. The evidence base relating to PCI is extensively reviewed in international guidelines and BCIS endorses the guidelines issued by the European Society of Cardiology (ESC) and the American Heart Association (AHA) and their updates. ${ }^{1} 2$ The guidelines presented here focus on issues pertinent to practice within the UK and set out a recommended template to ensure optimal delivery of patient care.

\section{STANDARDS \\ Institutional facilities}

A centre performing PCI requires at least one cardiac catheterisation laboratory. As there will be inevitable equipment failures-a second catheterisation laboratory is ideal to provide an uninterrupted service. In the absence of a functional second cardiac catheterisation laboratory-a non-cardiac radiological facility used for general radiology backup or a high resolution portable fluoroscopy unit with a small image intensifier is considered the minimum requirement.

Contemporary archiving in a Digital Imaging and Communications in Medicine compatible format is mandatory and it should be stored and accessible for a minimum of 8 years. Angiograms should be stored securely on a database allowing ready access 
during emergencies. PCI centres remote from surgical centres should have facilities for real-time image transfer to facilitate discussion and advice about individual patients' cases. Ideally clinical networks should invest in secure information technology that allows any neighbouring site in the region to view archived patient data as this would facilitate optimal emergency care.

Physiological measurements including accurate pressure recording and display of the waveforms on multiple simultaneous channels along with the facility to display and record a range of ECG lead configurations are required. Patients who have received sedation and/or analgesia should have their heart rate and oxygen saturation monitored continuously throughout the procedure.

Full resuscitation facilities including a defibrillator, intraaortic balloon counter-pulsation and an anaesthetic machine should be readily available in all catheterisation laboratories undertaking PCI. Facilities for monitoring anticoagulation (eg, activated clotting time) and blood gas analysis should be available within the catheterisation laboratory complex. A wide range of disposable angioplasty equipment including guide catheters, guide wires, balloons and stents must be available at all times and an appropriate method of inventory and stock control is mandatory. Availability of covered stents to treat perforation, pericardiocentesis sets to treat tamponade and an accessible echocardiography machine is mandatory. Recent technological advances have resulted in the availability of many additional tools including intravascular ultrasound (IVUS), optical coherence tomography (OCT), flow and pressure wires and equipment for laser and/or rotational atherectomy. The role of pressure wire in functional evaluation of coronary lesions is well established. This technology together with IVUS forms an essential part of interventional diagnostic procedures and should be available in catheter laboratories performing coronary interventions.

\section{Radiation protection}

All catheter lab staff should be aware of the latest guidelines on radiation protection. A representative of the radiation protection officer-usually a radiographer-should ensure that all staff comply with appropriate monitoring and that lead screens, aprons, glasses and other methods of radiation shielding are available and used appropriately. Monitoring of individual dose exposure is mandatory along with feedback of the cumulative dose.

Radiation risks from cardiac procedures should be discussed with the patient as part of the pre procedure consent process. ${ }^{3}$ It is good practice to include the hazards associated with radiation exposure on the consent form for PCIs. Almost all the current catheter lab radiography sources are equipped with methods to calculate and record peak skin dose and dose area product. Recording of the patient's dose exposure is mandatory and measures should be in place to recognise patients requiring multiple high dose exposures (eg, computed tomography pulmonary angiogram (CTPA) followed by PCI). The radiographer should notify the operator where radiation exposure is above the notification threshold (2000 mGy for first notification and subsequent notifications at every $500 \mathrm{mGy}$ increments ${ }^{3}$ ). The patient should be notified if the peak skin dose exceeds $3000 \mathrm{mGy}$ in order to highlight awareness of potential physical consequences. Many institutions have developed skin dose cards to notify the patient of the high dose exposure, which also details the warning symptoms and contact details for further advice. ${ }^{4}$

\section{Key points: institutional facilities}

- Two cardiac dedicated catheter laboratories are the minimal requirement for a $\mathrm{PCl}$ service undertaking emergency cases.

- Digital Imaging and Communications in Medicine archiving of images.

- Physiological assessment facilities in all interventional laboratories.

- Radiation protection mandatory.

\section{Institutional volume}

Although PCI could be considered as a routine procedure, it requires an experienced multidisciplinary team that can provide high quality care throughout the hospital stay. There has been extensive debate about what should be regarded as the minimum number of annual cases required for both institutions and individuals to maintain optimal performance. In the guidelines published in 2000, 200 procedures per annum was set as the standard for institutions, ${ }^{5}$ but since 2005 a minimum of 400 procedures per annum has been regarded as a minimum. ${ }^{6}$

These guidelines endorse and maintain the minimum centre volume of $>400$ procedures per annum. Recent NICOR data confirms the safety of these recommendations in current UK practice but BCIS recognises that this recommendation will require review with continued evolution of PCI techniques.

In 2013, 2 out of 98 National Health Service (NHS) units and $18 / 19$ private units in the UK were undertaking less than 200 procedures per annum. Twenty-two out of 98 were performing less than 400 procedures per annum. Centres performing fewer than 200 procedures per annum for three consecutive years have been named and contacted by BCIS. BCIS continues to recommend the same facilities and standards for both NHS and private hospitals with a minimum recommended volume of 400 interventional cases per year. In the future, every centre performing $<400$ cases per year will be contacted by BCIS who will notify NHS England and the Commissioners of sites whose practice is outside the recommended volume guideline.

\section{Interventional diagnostic procedures}

There are considerable data supporting the role of physiological assessment of stenosis using pressure wire prior to intervention. ${ }^{7-10}$ There is also data on the use of cross-sectional imaging with IVUS or OCT to measure lumen area and guide PCI. ${ }^{11}$ Use of pressure wire and use of IVUS/OCT are regarded as interventional diagnostic procedures by BCIS which endorses the use of these techniques in appropriate cases. Interventional diagnostic interventional cases should be performed in PCI centres with full interventional cardiology capability and by accredited interventional cardiologists able to treat any complications which might occur, for example, coronary dissection.

\section{Interventional team numbers}

The minimum recommended number of trained interventional cardiologists within a PCI centre is three. Some centres have used joint cover arrangements with neighbouring centres to facilitate the initiation of the service. This arrangement should be regarded as temporary and there is an expectation that a third local permanent colleague will be appointed within 2 years. 
PPCI for STEMI-PPCI

Specific standards required for centres that provide emergency PPCI for patients with STEMI have been developed by the Clinical Reference Groups. It is expected that Trusts should comply with all recommendations.

These BCIS guidelines recommend that patients undergoing PPCI should be treated in 24/7 PCI centres treating more than 300 PPCI patients per annum. In view of geographical isolation, this case volume may be impractical in some isolated areas but BCIS suggests that for the UK, PPCI centres should all perform an absolute minimum of 100 PPCI procedures per annum. Centres performing $<300$ PPCIs/annum should consider annually whether a Network approach which rationalises the number of adjacent PPCI centres would be a more appropriate model of care.

A PPCI centre should have two or more cardiac catheter laboratories and will require a larger catheter lab team to staff a manageable rota. PCI operators should regard their contribution to a PPCI rota as a fundamental part of their job plan. It is recognised that this will necessitate cooperation between trusts with regards to cross-charging, back-filling of duties at the base hospital and compensatory rest.

Ultimately a sustainable PPCI rota for Consultant Cardiologists should comprise a minimum of 6 Interventional Cardiologists and ideally 10.

\section{Key points: institutional volume}

- Minimum centre volume is 400 cases/year.

- Minimum of three interventional cardiologists per centre.

- $\mathrm{PPCl}$ centres should have at least two catheter laboratories and 24/7 provision of service for STEMI.

- PPCI centres should perform an absolute minimum of 100 STEMI/PPCI cases/year.

\section{Surgical cover standards}

BCIS fully supports the provision of PCI in appropriately selected patients in centres without on-site cardiac surgery, provided standard centre and operator requirements as described in this document are satisfied.

PCI without on-site cardiothoracic surgical cover is commonly performed in the UK. Emergency transfer for surgical intervention has come down significantly since the advent of intracoronary stents. While, $2.6 \%$ of patients undergoing PCI required emergency coronary artery bypass grafting (CABG) in $1991,{ }^{6}$ this has come down to $1.1 \%$ by 2007 and the latest BCIS audit data showed $0.05 \%$ patients required emergency transfer for surgical revascularisation in 2012. ${ }^{12}$ The eventual outcome in these patients would have been different without emergency surgical intervention.

Therefore, PCI centres without on-site surgical cover should have a viable protocol for emergency transfer to the nearest surgical centre. The protocol must be agreed by all stakeholders, including the relevant parties in the non-surgical centre and the surgical centre with which it works, local networks, commissioners, and the ambulance service. The protocol will need to address the training and availability of staff to accompany the patient, including an anaesthetist when required. Emergency transfer of patients should occur within a maximum of $1 \mathrm{~h}$, with the ability to start cardiopulmonary bypass within $2 \mathrm{~h}$ of the call for surgical intervention. ${ }^{13}$ Necessary equipment should also be considered, including a transportable intra-aortic balloon counter-pulsation pump (IABP). BCIS recommends that the feasibility of ambulance transfer of the IABP be tested to confirm it can be achieved within the required 120 min timeline. A good working relationship with the cardiac surgical team in the surgical centre is essential for all non-surgical PCI centres. It is considered good practice to undertake a virtual run (without the requirement for actual 'blue light' driving) of a catheter lab to surgical centre transfer with IABP annually.

\section{Off-site surgery}

In the UK the number and proportion of centres performing PCI without on-site surgery continues to increase year-on-year, and is now in the majority. In 2013 there were 69 non-surgical PCI centres and 48 surgical centres. In all 37008 PCI procedures were undertaken in units without on-site cardiac surgery, representing $40 \%$ of total UK activity. Within the current AHA/ American College of Cardiology PCI guidelines, published in 2011, there is a Class IIa recommendation for PPCI in hospitals without on-site surgery, whereas the guidelines give only Class IIb support for non-PPCI, stating that "Elective PCI might be considered in hospitals without on-site cardiac surgery, provided that appropriate planning for programme development has been accomplished and rigorous clinical and angiographic criteria are used for proper patient selection". ${ }^{14}$ In fact, since these guidelines were written, two major multicentre randomised controlled trials have been reported, and both support the safety of non-PPCI in hospitals without on-site cardiac surgery. In the CPORT-E trial, 18867 patients were randomised 3:1 to undergo PCI in centres with off-site surgery versus on-site surgery. ${ }^{15}$ There was no difference in the primary safety end point of 6 -week mortality $(0.9 \%$ vs $1.0 \%)$, nor in the incidence of emergency CABG $(0.1 \%$ vs $0.2 \%)$. Mortality in those patients who underwent emergency surgery was also no different between the non-surgical $(2 / 13,15.4 \%)$ and surgical $(2 / 10$, $20 \%$ ) centres. However, procedural success was lower in nonsurgical centres $(90.7 \%$ vs $91.4 \%, \mathrm{p}=0.007)$, and target vessel revascularisation was higher after 12 months $(6.5 \%$ vs $5.4 \%$, $\mathrm{p}=0.01$ ). More recently the MASS COM trial randomised 3691 patients undergoing non-PPCI 3:1 to be treated in non-surgical or surgical centres. ${ }^{16}$ Again there was no difference in the primary safety end point of 30-day major adverse cardiac events (MACE) $(9.2 \%$ vs $9.1 \%)$, nor in the need for emergency CABG $(0.3 \%$ vs $0.1 \%)$. In contrast to Cardiovascular Patient Outcomes Research Team-E trial (CPORT-E) there was also no difference in procedural success or in 12-month outcomes including target vessel revascularisation (TVR). It is difficult to translate the findings of these studies directly to UK practice given the substantial differences in the organisation of services, in particular very low volume non-surgical centres (117/year in MASS COM, 150/year in CPORT-E) but staffed, at least in CPORT-E, by operators all of whom were practising in surgical centres as well as nonsurgical centres, and whose individual PCI volumes were identical to those of the surgical centre interventionists. Nonetheless, they provide solid support for non-emergency PCI in centres without on-site surgery. Furthermore, a very large meta-analysis including 914288 patients undergoing non-PPCI in a number of European as well as North American centres also showed no difference in crude rates of inhospital mortality or emergency CABG between patients treated in hospitals with off-site surgery versus on-site surgery, although after adjustment for publication bias, the risk of inhospital death was reported to be higher by the investigators (OR 1.25, $\mathrm{p}=0.04){ }^{17}$ 


\section{Key points: surgical cover}

- Surgical cover arrangements must be annually confirmed and carefully documented.

- A maximum delay of 120 min from call to institution of cardiopulmonary bypass is the required standard.

- Annual virtual practice runs with IABP in situ are recommended practice.

\section{Staff standards}

Non-medical Cath Lab staff numbers and roles

The number of non-medical trained staff involved in a PCI procedure will depend on skill mix, complexity of the procedure and the number of medical staff involved. BCIS recognises the fundamental requirement of effective teamwork in optimising patient outcome and the multidisciplinary team is represented within BCIS by the Allied Professionals Working Group.

In most circumstances and in the absence of multiskilled practitioners, it is recommended that there is a minimum of two nurses per cath lab and one floater (one nurse at least band 5 and above) per shift for PCI procedures. In addition, one radiographer and one physiologist per lab (usually band 6 and above) would be considered normal practice. For units with more than one catheter lab, a separate additional coordinator should be considered (band 6 and above). Procedural roles are suggested below (table 1).

All staff should have a period of supervision and fulfil a competency-based training scheme within their hospital. There are currently no national competency guidelines for PCI specifically but documentation of completion of specified local training should be accessible. An Advanced Life Support certificate should be held by all senior members of the Cath Lab team. All other members of the team should hold a minimum of an intermediate life supoort (ILS) certificate and all be versed in emergencies within the Cath Lab. All members of the Cath Lab team should be familiar with the local rules and guidelines for radiation protection.

\section{Patient care and consent}

Patient preparation, informed consent and ward checklists should be performed as part of local standard operating policies.
Attention should also be paid to issues which may affect the patient's ability to lie comfortably during the case.

\section{Consent}

The General Medical Council and good clinical practice demands that patients are fully involved in the decisions about their care. Consequently an operator should be satisfied that the patient has been fully informed about the benefits and risks of any interventional procedure. Formal signing of the consent form may be delegated to a suitably trained and qualified person only if they have sufficient knowledge of the proposed procedure, and understand the risks involved. Patients must be fully involved in the decisions about their care including details of the procedure, benefits, procedural risks and likelihood of success. Ample time should be allocated to allow the patient to understand and discuss this information. All potential serious adverse outcomes must be explained including the possibility of death, acute myocardial infarction (MI), stroke, the requirement for emergency cardiac surgery and complications of vascular access. The likelihood of such events should be drawn from local and/or national statistics and will vary depending on the clinical scenario. The website http://www.gmc-uk.org/ guidance/ethical_guidance/consent_guidance_index.asp provides the Gemeral Medical Council (GMC) guidance 'Consent: patients and doctors making decisions together'.

Written consent is advised for all PCI procedures except in an emergency (1) when verbal consent may be taken together with full documentation in the case notes or when (2) it is either not possible to find out the patient's wishes or the patient lacks the capacity to give consent. Therefore, even in the setting of PPCI, some form of either written consent or postprocedural written record of consent is recommended.

A record of the consent form should be kept in the notes and given to the patient.

\section{WHO checklist}

In addition to ward-based checklists, the National Patient Safety Agency has asked all hospitals in England and Wales to implement a five-stage checklist for all interventional procedures based on the WHO safe surgery checklist (http://www. nrls.npsa.nhs.uk/resources/collections/10-for-2010/five-steps-tosafer-surgery/?entryid $45=92901$ ). Versions appropriate for catheter lab use include local adaptations, the interventional radiology checklist (approved by the National Patient Safety Agency) and a version endorsed by the BCS.

Table 1 Procedural roles of non-medical catheter lab staff

\begin{tabular}{|c|c|c|c|c|}
\hline Procedural roles & Nurse & Radiographer & Physiologist & Multiskilled practitioner \\
\hline Checklist/checking into lab & $x$ & $x$ & $x$ & $x$ \\
\hline Lead on safe surgery & $x$ & $x$ & $x$ & $x$ \\
\hline Scrub & $x$ & & & $x$ \\
\hline Circulate & $x$ & & & $x$ \\
\hline Monitor haemodynamics & $x$ & & $\mathrm{x}$ & $x$ \\
\hline Radiation protection & $x$ & $x$ & $x$ & $x$ \\
\hline Sedation/intravenous administration & $x$ & & & $x$ \\
\hline Cannulation & $x$ & $x$ & & $x$ \\
\hline Access site closure & $x$ & & & $x$ \\
\hline Infection control & $x$ & $x$ & $x$ & $x$ \\
\hline Documentation & $x$ & $x$ & $x$ & $x$ \\
\hline Handover & $x$ & & & $x$ \\
\hline Lab preparation/cleaning & $x$ & $x$ & $x$ & $x$ \\
\hline
\end{tabular}


The five stages are:

1. Team brief: All members of the team meet before the start of the list to discuss the requirements of the day's list. Safety issues such as patient allergies and comorbidities are discussed as well as potential procedural difficulties. Equipment that may be needed is discussed.

2. Sign in: On receipt of each patient into the cath lab, check identification, consent, allergies, pregnancy disclaimer (female 11-55 years), renal function, local preprocedure checklist. May be performed by a nurse, radiographer or physiologist.

3. Time out: Before start of each procedure (can be performed after draping, but before local anaesthetic) with all team members taking part including the operator. Involves further check of 'right patient/right procedure/right route', consent, allergies and safety for the team-including lead aprons and doors locked.

4. Sign Out: At the end of the procedure two practitioners ensure the procedure has been documented fully and detailed handover given including any recovery issues. Address safety issues including sharps counted, swabs counted, and identify any equipment problems/stock issues.

5. Debrief: At the end of the list, a discussion to identify good and learning points, answer questions from team members, promote learning and development of the team and discuss any incidents and identify prevention strategies.

\section{Analgesia/sedation}

The majority of PCI procedures can be safely performed without any sedation or under conscious sedation, administered without the need for an anaesthetist. This requires the patient to be responsive to verbal and tactile stimuli throughout the procedure. Each Catheter Lab should have a conscious sedation policy and competencies which should be achieved and signed off before any nurse/practitioner gives conscious sedation. Carbon dioxide levels may rise under conscious sedation, particularly when procedures are prolonged and supplementary oxygen is given in patients with lung disease or sleep apnoea. In these clinical situations, capnography and/or anaesthetic support should be considered.

\section{Postprocedural care}

After completion of the PCI procedure the patient is transferred to either a ward or recovery area. Full details of the procedure, details of the drugs administered during the procedure, and recommendations for drug therapy thereafter, together with complete procedural documentation should be handed over to the receiving area staff. Subsequent nursing care should include observation of the point of vascular access, and haemodynamic and ECG monitoring. A suggested scheme is included below (table 2).

Before discharge from hospital the patient with PCI should be given written instructions relating to possible vascular and other complications and advice on activity during the recovery period including driving, etc. Arrangements should include a schedule for return to work and proposed follow-up along with drug therapy instructions and planned interactions with formal cardiac rehabilitation programmes. Patient advice cards which outline the prescribed dual antiplatelet regime and a contact telephone number are valuable.

\section{Routine measurement of biomarker/cardiac enzymes after PCl}

In patients with acute coronary syndrome (ACS), biomarker elevation before the PCI procedure occurs regularly. Consequently interpretation of elevated biomarkers troponin, creatinine kinase myocardial band (CKMB) post procedure is difficult and measurement in ACS patients is not recommended. However, in patients with stable angina undergoing elective PCI measurement of biomarkers at an appropriate interval (4-6 h when troponin is being measured) after the procedure is valuable and this is considered to be best clinical practice.

\section{Key points: staff standards/patient care and consent}

- Minimum staffing levels: two nurses per catheter procedure.

- Operators should be satisfied that the patient has been fully informed about the benefits and risks of any interventional procedure. This must be confirmed with a written consent form or in the notes.

- The WHO checklist or a local equivalent is mandatory.

\section{Operators}

A successful interventional cardiologist requires a combination of manual dexterity and clinical judgement. Appropriate

Table 2 Postprocedural care, monitoring and observations

\begin{tabular}{|c|c|}
\hline & Radial \\
\hline $\begin{array}{l}\text { Elective } \\
\text { angiography }\end{array}$ & $\begin{array}{l}\text { Observations/access site check every } 15-30 \mathrm{~min} \\
\text { for } 1 \mathrm{st} \mathrm{h} \text {. Mobilise immediately if no sedation. } \\
\text { Discharge after } 2 \mathrm{~h}\end{array}$ \\
\hline Day case $\mathrm{PCl}$ & $\begin{array}{l}\text { No ECG monitoring unless stated. Single } \\
\text { 12-lead ECG post procedure. Observations/ } \\
\text { access site check every } 15-30 \text { min for } 1 \mathrm{st} h \text {, } \\
\text { then repeated at } 2 \mathrm{~h} \text {. Mobilise when sedation } \\
\text { worn off. Discharged at minimum } 4 \mathrm{~h}\end{array}$ \\
\hline $\begin{array}{l}\text { Complex } \mathrm{PCl}- \\
\text { elective }\end{array}$ & $\begin{array}{l}\text { Cardiac monitoring required, post procedure } \\
\text { 12-lead ECG. Observations and access site } \\
\text { check every } 15-30 \text { min for as long as required. } \\
\text { Mobilise when appropriate. Patients may require } \\
\text { overnight stay, discharged following day }\end{array}$ \\
\hline
\end{tabular}

Successful (without complication) using femoral access with device closure

Observations/access site check every 15-30 min for $1 \mathrm{st} h$. Mobilise after $30 \mathrm{~min}$. Discharged min 2-3 h

No ECG monitoring unless stated. Single 12-lead ECG. Observations/access site check every 15$30 \mathrm{~min}$ for $1 \mathrm{st} h$, then repeated at $2 \mathrm{~h}$. Mobilise $>60$ min and after sedation worn off. Discharge minimum $4 \mathrm{~h}$

Cardiac monitoring required, post procedure 12-lead ECG. Observations and access site check every 15-30 min for as long as required. Mobilise when appropriate. Patients may require overnight stay, discharged following day
Successful (without complication) using femoral access-manual haemostasis

Observations/access site check every $15-30$ min for $1 \mathrm{st} h$. Supine $1 \mathrm{~h}$, sitting $2 \mathrm{~h}$; mobilise $3 \mathrm{~h}$. Discharge min 3-4 $\mathrm{h}$

No ECG monitoring unless stated. Single 12-lead ECG. Arterial sheath removed ACT $<150$ s. Observations/access site check every 15-30 min pre and post sheath removal. Discharge minimum 5-6 $\mathrm{h}$

Cardiac monitoring required. 12-lead ECG post procedure. Arterial sheath removed when ACT $<150$ s. Observations and access site check every 15-30 min for as long as required. Patients may require overnight stay, discharged following day

Inpatient PCl Cardiac monitoring required for STEMI patients and patients with haemodynamic compromise during the procedure. Post procedure 12-lead ECG. It is (urgent NSTEMI/ recommended that access site and patient checked every 15-30 min for as long as required. Bed rest as per hospital protocol STEMI)

ACT, activated clotting time; NSTEMI, non-ST segment elevation myocardial infarction; PCl, percutaneous coronary intervention; STEMI, ST segment elevation myocardial infarction. 
emphasis is required on both the practicalities of the technique and the procedural numbers but also recognition of the role of overall patient assessment and balancing the risk-to-benefit ratio. It remains axiomatic that maintenance of the practical skills necessary for interventional cardiology requires the regular performance of procedures to maintain those skills. Debate is inevitable when setting an arbitrary minimum number of procedures required by an individual to maintain competence as an independent PCI operator. BCIS defines an independent PCI operator as an individual who can decide on PCI, plan the strategy and perform the case without consulting any other operator, buddy, mentor or trainer.

Previous guidelines recommend a minimum of 75 PCI procedures per year for independent operators. This did not include interventional diagnostic only procedures, which are defined as the use of Fractional Flow reserve (FFR), IVUS and OCT (and optical frequency domain imaging) when no PCI ensues. Although there is insufficient evidence to change the minimum number of total procedures as an appropriate ideal, it is recognised that interventional diagnostic procedures do use a subset of the practical skills needed for PCI. Consequently the recommended combined case volume over 2 years is a minimum of 150 cases which can include up to a maximum of 30 interventional diagnostic procedures (including a mix of elective and non-elective patients).

There is no specified minimum volume for operators undertaking PPCI. However it is recognised that these patients are among the most challenging patients and that familiarity with teamwork is essential. Consequently operators participating in PPCI cases should undertake an absolute minimum of $>50$ elective/emergency cases/annum within the emergency PPCI site and a total workload of at least 120 PCI cases plus up to 30 interventional diagnostic procedures.

For Consultants or independent Specialist registrar (SPR) operators who fulfil the guideline of 150 total procedures over 2 years and who then are absent from practice for less than 6 months-no additional training is required. If the period of absence exceeds 6 months and is less than 2 years a buddy system should be employed for 20-50 PCI procedures (proportional to the period of absence exceeding 6 months) before the operator becomes fully independent again. Appointment of the buddy should be agreed by the regional PCI training director.

Operators who have fully trained but have not undertaken any procedures for 2 years or more should perform at least 75 PCI procedures with a mentor. This designated mentor should formally assess the ability, aptitude and clinical judgement of the operator.

Cardiologists who have never been trained in PCI and wish to start PCI should undergo a full formal interventional training programme.

Individual cardiologists who undertake PCI on multiple sites are responsible for making sure that data from all the sites are sent to NICOR where the data can then be aggregated and provided back to them for appraisal and revalidation. ${ }^{18}$ Only one operator can ultimately be responsible for a PCI case although two Consultant Cardiologists may scrub together. It is recommended that it is clarified before the initiation of the case which consultant is responsible for the case and the outcome. In cases of debate it is recommended that the consultant with the lowest GMC number is recorded (this will be the physician registered longest with the GMC) as the default operator.

\section{Key points: operator standards}

- Diagnostic interventional cases are pressure wire/OCT (and optical frequency domain imaging) or IVUS cases.

- Total procedural volume for PCl operators within a 2-year period is a total of 150 cases (can include up to 30 diagnostic interventional cases).

- Operators participating in $\mathrm{PPCl}$ cases should be undertaking an absolute minimum of 50 elective/emergency cases/annum within the emergency PPCI site.

\section{The multidisciplinary team}

International guidelines recommend the utilisation of multidisciplinary teams to guide the management of patients with coronary artery disease. ${ }^{12}$ Careful discussion about the pros and cons of medical therapy versus revascularisation whether with surgery or PCI is accepted best practice. The detailed functioning of such a 'Heart Team' varies across the UK as does its composition, frequency and the type of cases discussed.

In 2013, the BCS, the BCIS and the Society for Cardiothoracic Surgery commissioned a joint working group. Its task was to produce a document with the intention of providing guidance as to the essential components of a Heart Team in terms of how it should be structured and how it should function. ${ }^{19}$ The complete document can be accessed on the websites of all three societies (http://www.bcis.org.uk, http://www.bcs.com and http://www. scts.org). This includes its attendance, scheduling and frequency as well as the type of cases to be discussed and the minimum data to be presented. Importantly, it also addresses the required managerial, administrative and technological support, systems of documentation, feedback and audit, and the degree and timing of patient/carer involvement.

Key areas include:

A. Patient and carer involvement

The patient's presence at the multidisciplinary team (MDT) meeting is usually impracticable. Thus a Clinician, who is familiar with the case and is able to represent the patient's best interests and any declared wishes, should attend. Facilitated by the coordinator, it should be that clinician's responsibility to ensure that the patient is advised of the MDT's recommendations. In elective cases, that transfer of information should be undertaken in an outpatient setting which will also present an opportunity to initiate the consenting process.

In cases of equipoise, that is, the evidence for one management strategy is balanced by that for another, this should be discussed with the patient in order to gauge their own preference. A Clinician of any of the specialties represented at the MDT should undertake that discussion emphasising the positive aspects of having more than one treatment available, rather than giving the impression of clinical indecision.

B. Clinical composition and frequency

The MDT should be chaired by a consultant and comprise a minimum of an interventional cardiologist, a cardiac surgeon and ideally a non-interventional cardiologist in order to be quorate.

A clinical individual who is familiar with the case, and is directly responsible for that patient's care, should present the data related to each patient to be discussed.

MDT meetings should be held at least once per week and at least $1 \mathrm{~h}$ should be allocated.

C. Facilities and technical considerations

MDT meetings should be held in a dedicated room that offers privacy and space for attendees. Patient imaging should be 
displayed on screens offering diagnostic medical quality and be visible to all attendees. IT support should ensure that images from remote centres can also be viewed and that where necessary two-way voice communication with referral units can be accomplished during the meeting. It should be available during the meeting if required in order to deal rapidly with any technical problems.

D. Administration and managerial support

A dedicated MDT coordinator should be present in order to (1) assimilate patient information prior to the MDT meeting, (2) document attendance; (3) record recommendations in each case, and (4) facilitate communication of that decision to all relevant parties.

In cases in which decision-making is not straightforward the essential elements of the discussion should also be recorded. The MDT recommendations, as well as any additional information where relevant, should be recorded by the coordinator and signed by the Chair.

E. The type and range of cases to be discussed

MDT discussion should be considered routine when diagnostic angiography reveals a left main stem stenosis or obstructive multivessel coronary disease, and there is no clinical mandate to proceed immediately to PCI. This is particularly relevant for patients in which revascularisation is considered high risk or highly complex including most patients with a chronic total occlusion. There may be occasions when clinical circumstances do not allow time for discussion in a formal MDT setting. In such cases ad hoc discussions will take place, the essence and outcome of which should be documented into the case notes.

F. Minimum data discussed and method of presentation

Clinical data, significant comorbidity and the results of all relevant investigations should be made available to the MDT coordinator prior to the meeting. Patient data should be presented to the MDT by the clinician familiar with the patient and their wishes with regards to possible management plans.

A recognised scoring system should be used in evaluating the procedural risk of either CABG or PCI. In addition the SYNTAX score could also be calculated and used in order to inform the discussion as to the burden of disease. ${ }^{20}$

G. MDT considerations and functioning in non-surgical centres The principles of MDT structure and functioning apply to nonsurgical cardiology units to the same extent as they do to cardiac surgical centres.

MDT meetings should occur with the same frequency and timing in non-surgical centres as in surgical units.

In order to ensure that all relevant specialties are represented, clinicians may have to travel between units to attend meetings. If this is considered to be impractical then teleconferencing technology is recommended and should be supported by the respective Trusts.

H. Timing and integration within job planning

MDT meetings should be scheduled during working hours.

Job planning for consultant staff should incorporate their attendance at MDT meetings.

I. Documentation, feedback and audit of outcomes

The outcome of MDT meetings and their recommendations in each case should be documented and signed by the Chair. In situations in which the discussion is not straightforward those particular elements that have led to the eventual recommendation should also be documented accurately and signed by the Chair. This applies particularly to cases that are judged to be at increased risk for either CABG or PCI. In addition to recommending the need and mode of revascularisation, the MDT should also indicate the priority in each case.
Units should put in place a system in which cases discussed in an MDT are followed up to ascertain whether the recommendations made were carried out. If there is any aberration then the reasons for this should be discussed and documented in the patient's medical records and then rediscussed at the MDT.

\section{Key points: MDT}

- The MDT should comprise a minimum of an interventional cardiologist, a cardiac surgeon and a non-interventional cardiologist in order to be quorate.

- A clinical individual who is familiar with the case, and is directly responsible for that patient's care, should present the data related to each patient to be discussed.

- Job planning for consultant staff should incorporate their attendance at MDT meetings.

\section{MONITORING STANDARDS}

\section{Audit}

All PCI centres are expected to collect comprehensive and accurate data that relates to the interventional treatment they provide for their patients. This includes information pertaining to the structure of service provision, the appropriateness of intervention, and the process and outcomes of PCI. The data are expected to relate to each PCI centre (and not combinations of centres even if within a single trust).

BCIS provides a clinical data set to allow a national comparison of results of interventional techniques and comparative audit. ${ }^{21}$ This data set is updated periodically to remain pertinent to current therapeutic strategies. Structural changes to the data set are made as infrequently as possible to minimise disruption to the data collection infrastructure, but more frequent updates are made to the libraries of device names and medications. It is expected that changes to the BCIS data set can be implemented within a notice period of 3 months. Definitions of the variables are contained within the data set document which takes the form of a spreadsheet and is available on the BCIS website. BCIS oversees and guides the collection and analysis of these data which is currently hosted by the NICOR.

It is the responsibility of the Trust/Hospital to provide the appropriate audit and information technology infrastructure to allow clinicians assisted by data managers to collect comprehensive and accurate data and to achieve data submission that complies with deadlines set by BCIS and NICOR to permit data analysis and presentation within the required timescales each year.

Each cardiology department should provide the name of a designated clinician to lead the audit process and ensure that the infrastructure is in place. However, the ultimate responsibility for data completeness and accuracy rests with the consultant PCI operator responsible for the PCI procedure.

External data validation is not currently possible. It is therefore recommended that regular internal validation of case ascertainment, data completeness and data accuracy is performed. This is particularly recommended for the date/time fields for patients treated by PPCI and in the adjudication of high risk clinical features. An annual internal review of cardiogenic shock outcomes and data entry is especially recommended to ensure consistency and accuracy.

The BCIS data set requires that information be collected from symptom presentation through to discharge from that PCI 
centre. Systems to collate and record events, particularly those that occur after a patient leaves the interventional lab require organisation and funding. Audit to ensure the completeness and accuracy of these processes should be performed locally. Outcome data which pertains to catheterisation laboratory discharge alone is insufficient.

Recommendations for data collection and submission apply to NHS and private centres.

\section{Institutional audit and clinical governance}

BCIS reports the results of an analysis of UK-wide PCI activity on an annual basis. The information is made available on the BCIS website for download. These data can be used by individual PCI centres to assess trends and to benchmark their own activity. Internal presentation of a PCI centre's outcomes and their relative performance against the national data is recommended on an annual basis.

In addition it is recommended that there is regular review of each PCI centre's practice against established national and international guidelines including National Institute for Health and Care Excellence (NICE), the ESC and AHA as deemed applicable to UK practice. Regular discussions should include individual case presentations for all unexpected mortality and morbidity. Additional audit of other complications and, for example, complex patient or complex lesion subsets are recommended. It is expected that Trusts will provide appropriate support for this process of clinical governance to allow collation and analysis of these data, ring fenced time for audit meetings, facilities for presentation, and support so that the meeting outcomes can be formally minuted and these minutes archived.

The specifics of the practice of institutional audit vary considerably around the country. Examples of good practice include random review of individual cases by randomly allocated colleagues and presentation of the case.

\section{Individual operator outcomes}

BCIS in collaboration with NICOR, will provide all individual operators with a detailed breakdown of their own PCI activity that includes process control charts and risk-adjusted outcome analysis. The aim is to provide data for each PCI operator that can be used in the process of annual appraisal and revalidation.

\section{Public reporting}

BCIS, in collaboration with NICOR, will provide a set of analyses at institutional and individual operator levels intended to be understood by the general public. This process started in 2013, with the intention of providing 3 -year rolling data in the future. Overall numbers of PCI procedures, a split by clinical syndrome and risk-adjusted outcomes will be presented in the first instance.

Key points: monitoring institutional standards

- All PCl centres are expected to collect comprehensive and accurate data that relate to the interventional treatment they provide for their patients.

- Regular departmental discussions should include individual case presentations for all unexpected mortality and morbidity.

- BCIS will provide operators with a detailed breakdown of their own PCl activity that includes risk-adjusted outcome analysis.

\section{ANNUAL APPRAISAL AND REVALIDATION}

The results of individual operators are likely to be improved by sharing experiences with colleagues. Discussion between operators, both formal and informal, should be part of departmental meetings. Maintaining optimal clinical practice in coronary intervention requires ongoing review of the medical literature. An awareness of the evolution of technology and the availability of adjunctive pharmacology is mandatory.

BCIS recommends a minimum of 4 days a year attending national and international meetings relating to cardiovascular intervention. A record of Continued Professional Development using the Royal College of Physicians (RCP) website is recommended. Demonstration of completion of local statutory mandatory training along with demonstrable participation in quality improvement process should be considered standard and be presented within the revalidation portfolio.

BCIS will provide their members who are individual operators with a detailed breakdown of their own PCI activity to be used in the process of annual appraisal and revalidation.

\section{NEW CENTRES}

The development of a new PCI site should be agreed by Hospital Trusts with all the relevant stakeholders through a strategic plan developed with the local cardiac network. Stakeholders include the local surgical centre, other nearby PCI centres, local commissioners of healthcare and specialist commissioners. Recommendations for the structure of a new PCI service are available on the BCIS website and should be used as a guide. ${ }^{22}$

Once a service is prepared for clinical commissioning a request should be made to the secretary of BCIS by the Chief Executive of the Trust. This request should include a statement confirming that the proposed PCI service will not begin until BCIS approval for service initiation is obtained. The BCIS peer review team will comprise two Interventional Cardiologists from outside the region who will arrange a mutually convenient time for the visit. It is expected that the visit will be arranged within 8 weeks of the request and the BCIS team will expect all of the documentation outlined on the website to be available prior to the visit.

At the completion of the visit the BCIS representatives will write a report and submit it through the Clinical Standards Group to BCIS Council. This report is then returned to the Trust Chief Executive with copies to the relevant Network and NHS England.

Follow-up visits are not anticipated subsequent to the agreement about the initiation of the service unless special circumstances apply.

\section{TEACHING AND TRAINING}

A training programme must ensure that its trainees acquire a sound knowledge base of the basic principles that underpin the practice of interventional cardiology. It is recommended that training programmes have a core curriculum that covers at least the following important topics:

- Anatomy and physiology pertaining to the cardiovascular system.

- In particular the trainee needs to understand coronary anatomy including its variations and congenital abnormalities, cardiac haemodynamic function and coronary physiology.

- Pathophysiology of cardiovascular disease.

- Pharmacology principles to provide an understanding of cardiovascular contrast agents and drugs commonly used for invasive procedures. 
- Principles of haemostasis including indications for and complications of vascular closure devices.

- Radiology imaging and radiation safety. This should include design and operation of X-ray cine angiographic units, basic radiation physics, radiation quality assurance, and an understanding of the biological risks from radiation exposure so as to minimise radiation exposure to the patient and catheterisation laboratory staff.

- Knowledge of lesion assessment with quantitative coronary angiogram analysis, physiological assessment and intracoronary imaging.

- The design and performance of interventional devices.

- Clinical management strategies to include case selection, performance of the procedures, and subsequent management of the patient before discharge and beyond. A clear understanding of the indications, limitations, and complications of the procedures must be acquired.

\section{Training in cardiac catheterisation}

Before undertaking training in interventional cardiology the trainee must achieve competence in general cardiology and in diagnostic cardiac catheterisation. The trainee should have completed training in diagnostic cardiac catheterisation procedures as a primary operator. These should include left heart catheterisation and coronary arteriography, as well as graft studies. Before formal training in intervention begins, the trainee is expected to have assisted in at least $25 \mathrm{PCI}$ procedures. Specialist training should be the focus of the last 2 years in the SpR training programme.

\section{Selection}

Training institutions should have in place a method of formal assessment and selection for the training places that can withstand fair scrutiny. The process of selection of an interventional trainee differs between institutions with no agreed mechanism nationally. Competitive interviews are recommended. After the selection, ongoing formal assessment throughout the period of training is mandatory with clear bidirectional feedback.

If it is considered that training would be appropriate for an individual but local circumstances are such that the training cannot be provided locally, an interdeanery transfer can be considered.

Local arrangements with neighbouring centres should be encouraged for trainees to be able to train in techniques not available at the host institution.

\section{Procedural numbers and scope}

Defining the minimum number of PCI procedures that constitutes an acceptable training in PCI remains difficult. A minimum of 200 procedures/year over the 2 years prior to consultant appointment with at least 125 as the first operator is the minimum requirement. During these cases the trainee must be fully involved in preprocedural evaluation of the patient, selection of equipment, discussion of risk and benefits and the outcomes of each intervention. The trainee should be directly involved in the reporting of the procedure including completion of database archiving, and documentation of any complications up to the time the patient is discharged from hospital. A review of the trainee's experience and outcomes should be performed on a regular basis.

Practical skills should be assessed on a competency basis. Completion of a logbook is mandatory for practical procedures along with completion of existing educational assessments. Observations by the educational supervisor and by a consultant other than the educational supervisor will be part of the assessment methods.
The training should have the following components:

- Core procedures including vascular access and vascular access management-including both radial approach and femoral approach.

- Conventional balloon angioplasty.

- Coronary artery stenting.

- PCI in the setting of ACS including primary intervention for STEMI.

- Pericardial aspiration.

- Intra-aortic balloon counterpulsation and other techniques of mechanical circulatory support.

- Intracoronary imaging.

- Coronary flow measurements/pressure wire measurements.

- Use of protection devices.

- Use of techniques to treat coronary perforation.

Experience in other techniques would depend on the expertise and practice of the institution. These could include post-Cetrificate of Completion of Training (CCT) training in the following:

- Rotational atherectomy

- Embolisation devices (eg, coils)

- Intravascular foreign body retrieval

- Adult structural heart intervention including:

- Balloon valvuloplasty.

- Transcatheter aortic valve implantation.

- Transcatheter closure of congenital/acquired defects.

- Left atrial appendage occlusion.

For the purpose of training it should be left to the trainer to determine when a trainee has been the principal (first) operator. The trainee must personally perform the procedures under the direct supervision of a recognised trainer. The trainer who takes overall responsibility for the patient must be immediately available in the catheterisation laboratory to supervise the trainee.

Trainees should be exposed to a comprehensive range of patient mix and these should include patients with stable angina and ACS such as unstable angina, acute MI and cardiogenic shock. All trainees should be able to acquire significant experience in the management of patients who require circulatory support, particularly with intra-aortic balloon counterpulsation.

Trainees must be familiar with the BCIS/ Central Cardiac Audit Database (CCAD) audit requirements for PCI and be aware of and participate in data collection and validation.

\section{Education}

BCIS recommends that an interventional training programme should hold a regular interventional meeting. This should address the core curriculum subject matter and should provide an opportunity to review both the diagnostic and interventional cases with respect to patient selection, the performance of the procedure, clinical outcome and any complications. The MDT meeting as an integral part of decision making should have active involvement of the interventional trainees.

Participation in clinical research by the trainees should be a core requirement for the programme. Trainees should be encouraged to initiate individual research and participate in trials in which the institution is taking part. Trainees should be active participants in data analysis and presentations and be encouraged to undertake clinical audit and follow-up of patients.

Trainees should spend at least 4 days each year attending appropriate educational meetings. Participation in the BCIS Basic Cardiovascular Intervention Course, The Advanced Cardiovascular Intervention Course for Trainees and annually the Advanced Cardiovascular Intervention are strongly recommended. Further relevant opportunities for education come in the form of 
international Fellows Courses, and the two major international meetings (Euro-PCR and Transcatheter Therapeutics meeting (TCT)).

\section{Trainers}

Each institution with an interventional training programme should have at least three experienced clinical Interventional Cardiologists who have each performed a minimum of 500 procedures in their careers. Each centre will have a designated training programme Director who will ensure trainee selection, appraisal and assessment. The programme director should have a career experience of a minimum of 1000 coronary interventional procedures. An interventional training centre will perform a minimum of 400 PCI procedures a year to allow a trainee to participate in the full spectrum of coronary intervention. We recommend that the number of trainees accepted into a training programme should reflect the institutional volume and the number of senior trainers available. Generally a PCI programme should be an integral component of a comprehensive service and should have on-site capabilities including a coronary care unit, cardiac surgery, cardiac intensive care and cardiac imaging including echocardiography, cardiac magnetic resonance scanning and nuclear cardiology.

High volume PCI programmes without on-site cardiac surgery provide excellent PCI training and training in these centres should be considered as an equivalent to training in a surgical centre. However it is expected that trainees will spend time training within a surgical centre, and that arrangements are made to allow interaction with neighbouring on-site surgical centre interventional trainers, trainees and cardiac surgeons.

\section{Trainee evaluation}

The responsibility for trainee evaluation should reside with the Programme Director for Education. Regular review of the trainee's feedback along with trainer's feedback, and presentation of that within the Deanery should occur on an annual basis. The programme director should be responsible for confirming that trainees have completed their interventional training satisfactorily as required by the core training requirements.

\section{FUTURE DEVELOPMENTS Commissioning}

The RCP/BCS response to the NHS White Paper Equity and excellence: liberating the $\mathrm{NHS},{ }^{23}$ is endorsed by BCIS including liaison at local vascular/chronic disease management board level between the local cardiology service and primary care clinical commissioning groups. The new Cardiovascular Disease Outcomes Strategy 2013 emphasises agreed care pathways for cardiac conditions across the local health economy. ${ }^{24} \mathrm{NHS}$ England and clinical commissioning groups have committed to reviewing, through the specialty's clinical reference group and local senates/networks, achievements in national cardiac audits including the NICOR, together with local mortality trends, in setting its priories.

Cardiovascular networks are recognised to have an important relationship with commissioning and PCI has shown the efficacy of that in the roll-out of network-based PPCI. Service level agreements should reflect expected outcomes to meet national cardiac audit outcomes. These key points for commissioners have been taken from the RCP's Consultant physicians working with patients: The duties, responsibilities and practice of physicians in medicine. ${ }^{25}$ Further information on service organisation, workforce requirements and job plans for cardiology services can also be found in this document.

\section{New technology}

Interventional cardiologists use and manage a very wide range of medicines and medical devices. Within the UK, the MHRA is responsible for regulating all medicines and medical devices by ensuring their safety and efficacy. The MHRA is a centre of the Medicines and Healthcare Products Regulatory Agency which also includes the National Institute for Biological Standards and Control (http://www.nibsc.org/) and the Clinical Practice Research Datalink (http://www.cprd.com/). The MHRA is an executive agency of the Department of Health. Its role includes guidance, safety alerts and links to educational material to assist cardiology practitioners in the safe use and management of medicines and medical devices.

NICE provides national guidance and advice to improve health and social care. NICE was originally set up in 1999 as the National Institute for Clinical Excellence, and in 2005, after merging with the Health Development Agency, the name changed to the National Institute for Health and Clinical Excellence. At this time NICE took on responsibility for developing guidance and quality standards through the work of independent committees. NICE currently provides guidelines, technology appraisals, guidance on interventional procedures, new medical technologies and use of diagnostic techniques. These currently include guidelines on the use of drug-eluting stents for patients with coronary artery disease.

\section{Research}

Research is a fundamental part of interventional cardiology and its scope goes beyond the limits of this document. The UK has a prominent role in interventional research and this is important for patients, interventional teams and the broader NHS. BCIS supports research in a number of ways which include the R\&D group which has as its primary goal the promotion of interventional research in the UK. The core philosophy is that the best research is planned, conducted and reported by motivated teams that 'own' the project. The BCIS R\&D committee seeks to facilitate the regular performance of quality, perhaps collaborative, studies in centres that have not previously regarded this as core business, or who have perhaps been overlooked when research organisations select partners.

BCIS also encourages the use of the BCIS audit data set for observational research, and this has resulted in a number of publications. Applications to use the data set for research can be made via the NICOR website.

Within the UK the National Institute for Health Research Clinical Research Network (NIHR CRN) provides health service infrastructure (eg, research support staff such as clinical research nurses; and research support services such as pharmacy, pathology and radiology) to support clinical research in the NHS in England. The NIHR CRN comprises 15 local CRNs and a national coordinating centre whose purpose is to provide efficient and effective support for the initiation and delivery of funded research in the NHS. Some of this research is funded by the NIHR, but most of it is funded by NHS non-commercial partners and industry. This activity makes an important contribution to improve the health of the population and to support economic growth; and the NIHR CRN features in the government's Strategy for UK Life Sciences.

The NIHR CRN allocates and manages funding to meet NHS support (eg, additional nursing time; pathology sessions; lab costs; imaging; additional outpatients costs) for eligible studies. 
These comprise randomised controlled clinical trials of interventions (including prevention, diagnosis, treatment and care) and other high quality well designed studies. The website http:// www.crncc.nihr.ac.uk provides the criteria governing eligibility of studies for Network support.

\section{BCIS meetings and live cases}

BCIS has a major role in training and education, with annual Society meetings and contributions to other national and international forums. These meetings may include lectures and debates, case-based discussions, angiogram review sessions, and live case demonstrations to exhibit procedural techniques and new technologies. Specifically BCIS provides two specialist interventional training courses/year together with an annual Advanced Cradiac intervention (ACI) meeting and a rotating autumn meeting.

Audiences at these meetings may include medical and nonmedical clinicians; members of the medical technology, device and pharmaceutical industries; members of government agencies or other professions; patients and their representatives; and members of the general public. Presentations may also include discussion of complications of cardiac interventions that cause morbidity and mortality. Critical review of these cases is an essential component of audit and education, and should be conducted in a supportive atmosphere to ensure that colleagues remain willing to present failures as well as successes. Attendance and participation by interventionalists at these meetings is very strongly encouraged and is a fundamental part of the revalidation process.

BCIS wishes to ensure that participants in all of these activities adhere to the highest standards of professional practice and that the care and dignity of patients is maintained. The link http:// www.bcis.org.uk/resources/C28_BCISRecommendationFPB provides the guidelines for the conduct of BCIS members at meeting and for the conduct of live cases.

\section{Author affiliations}

${ }^{1}$ Oxford Heart Centre, Oxford University Hospitals

${ }^{2}$ Department of Cardiology, Bristol Heart Institute Bristol, Bristol, UK

${ }^{3}$ Department of Cardiology, Leeds General Infirmary, Leeds, UK

${ }^{4}$ Southampton University Hospital

${ }^{5}$ Royal Cornwall Hospital Trust Cornwall, Cornwall, UK

${ }^{6}$ UK Manchester Heart Centre Central Manchester University Hospitals NHS Foundation Trust, Manchester University

${ }^{7}$ Queen Elizabeth Hospital Birmingham

${ }^{8}$ The Heart and Lung Centre, Royal Wolverhampton Hospital Trust, Wolverhampton, UK

${ }^{9}$ Cardiology Department, James Cook University Hospital, Middlesbrough, UK

${ }^{10}$ University Hospital of North Staffordshire, Staffordshire, UK

${ }^{11}$ Guys and St Thomas NHS Trust, London, UK

Acknowledgements BCIS is grateful to Ellie Gudde, Senior Sister Essex Cardiothoracic Centre, Sarah Carson, Catheter Laboratory Manager, Bristol Royal Infirmary, Catherine Theron, Unit Manager, Morriston Hospital and Dr Rod Stables, Interventional Cardiologist in Liverpool for their assistance with the preparation of this document.

Contributors The document is a consensus document, and each of the authors have been involved in the concept, writing, reviewing and submission.

Competing interests APB has received a grant to establish an Oxford-based training fellowship from Boston Scientific. DB is a paid proctor by Boston Scientific and Medtronic Corp.

Provenance and peer review Not commissioned; externally peer reviewed.

Open Access This is an Open Access article distributed in accordance with the Creative Commons Attribution Non Commercial (CC BY-NC 4.0) license, which permits others to distribute, remix, adapt, build upon this work non-commercially, and license their derivative works on different terms, provided the original work is properly cited and the use is non-commercial. See: http://creativecommons.org/ licenses/by-nc/4.0/

\section{REFERENCES}

1 Windecker S, Kolh P, Alfonso F, et al. 2014 ESC/EACTS Guidelines on myocardial revascularization: the Task Force on Myocardial Revascularization of the European Society of Cardiology (ESC) and the European Association for Cardio-Thoracic Surgery (EACTS)Developed with the special contribution of the European Association of Percutaneous Cardiovascular Interventions (EAPCI). Eur Heart $J$ 2014;35:2541-619.

2 Nallamothu BK, Tommaso CL, Anderson HV, et al. ACC/AHA/SCAl/AMA-Convened PCPI/NCQA 2013 Performance Measures for Adults Undergoing Percutaneous Coronary Intervention: a Report of the American College of Cardiology/American Heart Association Task Force on Performance Measures, the Society for Cardiovascular Angiography and Interventions, the American Medical Association-Convened Physician Consortium for Performance Improvement, and the National Committee for Quality Assurance. Circulation 2014;129:926-49.

3 Proceedings from the conference of radiation protection officers. London 2010.

4 North west radiation protection group, Skin card. AHP group BCIS website.

5 Coronary angioplasty: guidelines for good practice and training. Joint working group on coronary angioplasty of the British Cardiac Society and British Cardiovascular Intervention Society. Heart 2000;83:224-35.

6 Dawkins KD, Gershlick T, de Belder M, et al. Percutaneous coronary intervention: recommendations for good practice and training. Heart 2005;91(Suppl 6):vi1-27.

7 Pijls NH, van Schaardenburgh P, Manoharan G, et al. Percutaneous coronary intervention of functionally nonsignificant stenosis: 5-year follow-up of the DEFER Study. J Am Coll Cardiol 2007;49:2105-11.

8 Tonino PA, De Bruyne B, Pijls NH, et al. Fractional flow reserve versus angiography for guiding percutaneous coronary intervention. $N$ Engl J Med 2009;360:213-24.

9 De Bruyne B, Pijls NH, Kalesan B, et al. Fractional flow reserve-guided PCl versus medical therapy in stable coronary disease. $N$ Engl J Med 2012;367:991-1001.

10 De Bruyne $\mathrm{B}$, Fearon WF, Pijls $\mathrm{NH}$, et al. Fractional flow reserve-guided $\mathrm{PCI}$ for stable coronary artery disease. N Engl J Med 2014;371:1208-17.

11 Tearney GJ, Regar E, Akasaka T, et al. Consensus standards for acquisition, measurement, and reporting of intravascular optical coherence tomography studies: a report from the International Working Group for Intravascular Optical Coherence Tomography Standardization and Validation. J Am Coll Cardiol 2012;59:1058-72.

12 http://www.bcis.org.uk/pages/page_box_contents.asp?pageid=780\&navcatid=11 (accessed 17 Jan 2015).

13 Dehmer GJ, Blankenship JC, Cilingiroglu M, et al. SCAI/ACC/AHA Expert Consensus Document: 2014 update on percutaneous coronary intervention without on-site surgical backup. J Am Coll Cardiol 2014;63:2624-41.

14 Levine GN, Bates ER, Blankenship JC, et al. 2011 ACCF/AHA/SCAI Guideline for Percutaneous Coronary Intervention. A report of the American College of Cardiology Foundation/American Heart Association Task Force on Practice Guidelines and the Society for Cardiovascular Angiography and Interventions. J Am Coll Cardiol 2011;58:e44-122.

15 Aversano T, Lemmon CC, Liu L. Outcomes of PCl at hospitals with or without on-site cardiac surgery. N Engl J Med 2012;366:1792-802.

16 Jacobs AK, Normand SL, Massaro JM, et al. Nonemergency PCl at hospitals with or without on-site cardiac surgery. N Engl J Med 2013;368:1498-508.

17 Singh $M$, Holmes DR Jr, Dehmer GJ, et al. Percutaneous coronary intervention at centers with and without on-site surgery: a meta-analysis. JAMA 2011;306:2487-94.

18 http://www.bcis.org.uk/pages/page_box_contents.asp?PagelD=736 (accessed 22 Dec 2014).

19 http://www.bcis.org.uk/resources/MDT_joint_document_final_version.pdf (accessed 23 Dec 2014).

20 Sianos G, Morel MA, Kappetein AP, et al. The SYNTAX Score: an angiographic tool grading the complexity of coronary artery disease. Eurolntervention 2005;1:219-27.

21 http://www.bcis.org.uk/pages/page_box_contents.asp?pageid=697\&navcatid=11 (accessed 22 Dec 2014).

22 http://www.bcis.org.uk/pages/page_box_contents.asp?pageid=678\&navcatid=25 (accessed 23 Dec 2014)

23 https://www.gov.uk/government/uploads/system/uploads/attachment_data/file/ 213823/dh_117794.pdf (accessed 23 Dec 2014).

24 https://www.gov.uk/government/uploads/system/uploads/attachment_data/file/ 217118/9387-2900853-CVD-Outcomes_web1.pdf (accessed 23 Dec 2014)

25 https://www.rcplondon.ac.uk/sites/default/files/consultant_physicians_revised_5th_ ed_full_text_final.pdf (accessed 23 Dec 2014).

26 Athappan G, Patvardhan E, Tuzcu ME, et al. Left main coronary artery stenosis: a meta-analysis of drug-eluting stents versus coronary artery bypass grafting. JACC Cardiovasc Interv 2013;6:1219-30.

27 Serruys PW, Morice MC, Kappetein AP, et al. Percutaneous coronary intervention versus coronary-artery bypass grafting for severe coronary artery disease. $N$ Engl J Med 2009;360:961-72. 
28 Kahn JK. Angiographic suitability for catheter revascularization of total coronary occlusions in patients from a community hospital setting. Am Heart J 1993;126:561-4.

29 Koolen J, on behalf of the SYNTAX investigators. Optimal revascularization strategies in patients with total occlusions: 1 year results from the Syntax Trial. Presented at Japanese Circulation Society meeting 2009. 2009.

30 Werner G. Updates from the Euro CTO club. Presented at the Chronic Total Occlusion and Left Main Summit. 2013.

\section{APPENDIX 1: LEFT MAIN STEM DISEASE}

There is a very extensive literature of observational and randomised trials that have evaluated revascularisation in patients with left main disease. This literature has been extensively summarised in the 2014 ESC and the 2011 ACCF/AHA/SCAI guidelines. 114

In the pre percutaneous coronary intervention (PCI) era, coronary artery bypass grafting was the only option available for revascularisation in patients with significant left main disease. Subgroup analyses from the CASS registry and meta-analysis of several randomised controlled trials (enrolling a total of $>2500$ patients) indicated that, when compared with what we would now consider to be inadequate medical therapy, coronary artery bypass grafting (CABG) significantly improved survival in these patients. Multiple trials have reported on outcomes comparing CABG and PCI for the treatment of left main coronary disease. A recent meta-analysis of over 14000 patients enrolled in comparative trials of the two techniques reported no significant difference for all-cause mortality over a 5 -year follow-up period. ${ }^{26}$ Recurrent ischaemia and repeat revascularisation procedures were significantly more common in patients undergoing PCI for left main disease compared with CABG. The occurrence of stroke was less frequent in patients treated with PCI.

The most reliable contemporary data comparing PCI with drug-eluting stents or CABG for left main coronary disease is provided by the SYNTAX trial. ${ }^{27}$ In SYNTAX, 705 of the 1800 patients randomised had left main coronary disease. When the patients were grouped according to a measure of the complexity and extent of their coronary disease, using the SYNTAX score, a clear pattern emerges. In patients with less complex disease (low or intermediate SYNTAX score) CABG confers no survival advantage over PCI. For patients with more complex disease (manifest as a SYNTAX score greater than 33) CABG reduces mortality by a considerable extent.

Performing PCI in the left main stem should always be regarded as a challenging procedure. Operators and their catheterisation laboratory teams need to be experienced and proficient in rapidly responding to a sudden unplanned deterioration and the requirement for complex bailout stenting strategies. In some circumstances, rapid and effective deployment of haemodynamic support devices can be life-saving. It is important that these are immediately available and can be inserted and activated rapidly

Adherence to these standards will provide the best chance of a good patient outcome. The documented outcomes reported in the literature have been obtained by expert PCI teams. In circumstances when these minimal standards cannot be met, alternative referral is advised to a suitably equipped and experienced interventional colleague/centre. Preprocedural planning in left main stem cases requires multidisciplinary team (MDT) discussion and then careful evaluation, and this usually extends beyond the basic information provided by angiography alone. Preprocedure including intravascular ultrasound (IVUS) and/or measurement of fractional flow reserve is recommended to characterise the anatomical and haemodynamic features of the lesion, and guide stent sizing and procedural technique. There is
31 Joyal D, Afilalo J, Rinfret S. Effectiveness of recanalization of chronic total occlusions: a systematic review and meta-analysis. Am Heart J 2010;160:179-87.

32 Morino $\mathrm{Y}$, Abe M, Morimoto T, et al. Predicting successful guidewire crossing through chronic total occlusion of native coronary lesions within 30 minutes: the J-CTO (Multicenter CTO Registry in Japan) score as a difficulty grading and time assessment tool. JACC Cardiovasc Interv 2011;4:213-21.

often considerable difficulty in optimally sizing stents, and IVUS or optical coherence tomography should be performed after stent deployment to ensure that a good result with optimal stent apposition has been obtained.

In many patients with left main disease coronary calcification is common and the potential for adjunctive plaque modification to facilitate optimal stent implantation is high. This may not always be readily apparent on angiography, further emphasising the value of IVUS for preprocedure planning. Centres and operators performing left main intervention should ensure that they are proficient in the use of rotational atherectomy, and that this is available when performing urgent/elective PCI to the left main coronary.

\section{Key points: left main coronary intervention}

- When patients present with coronary instability associated with a culprit lesion in the left main, reduced antegrade coronary flow and clinical instability, PCl should be considered if the patient's clinical condition precludes performing CABG.

- In more stable patients, discussion by a multidisciplinary team is the recommended way of selecting an optimal revascularisation strategy.

- Availability of intra-aortic balloon counter-pulsation pump (IABP), IVUS, rotational atherectomy, and pressure wire systems are mandatory for left main intervention procedures along with the necessary expertise.

\section{APPENDIX 2: CHRONIC TOTAL OCCLUSIONS}

Chronic total occlusions (CTOs) are identified in up to a third of coronary angiograms ${ }^{28}$ but are relatively under-represented in percutaneous coronary intervention (PCI) practice. CTOs were approached in only 5\% of all PCIs and in $15 \%$ of stable cases in the UK in $2012 .{ }^{12}$ Reasons for this imbalance may include lower PCI procedural success rates, perceived or real superiority of surgical revascularisation, or an assumption that medical therapy alone is the only option for all but the simplest CTO lesion. However, surgical success is not assured for CTOs-only 68\% of CTOs were successfully bypassed in the Syntax Trial ${ }^{29}$ and success rates for PCI of $85 \%$ have been reported by CTO operators in Europe. ${ }^{30}$

High reported success rates for CTO PCI are likely to be related to development of more complex techniques, such as the retrograde approach and dissection/re-entry, in addition to improvements in specific procedural tools, for example, dedicated CTO wires, microcatheters (eg, Corsair, Tornus) and specialised re-entry equipment (Crossboss catheter, stingray wire and stingray balloon). For complex cases and techniques, it seems highly likely that case volume will have a part to play in predicting success.

Although observational data exists suggesting prognostic gain from CTO $\mathrm{PCI}^{31}$ no randomised trial data exist which 
demonstrate improvement in prognosis. The primary indication for CTO PCI therefore remains symptomatic improvement. Patients should be established on optimal medical therapy before being scheduled for PCI, but the bar need not be set higher than for other patients with stable angina and there is no requirement for multiple antianginals in maximum doses before PCI is considered.

Ad hoc PCI for CTOs should not be performed. It is recommended that angiograms in which a CTO is identified are presented to a multidisciplinary team (MDT). This should act as a safeguard against inappropriate conservatism and ensure that the most appropriate form of revascularisation is selected according to patient and anatomical factors.

Information presented to the MDT should include: symptom burden; current medical therapy; results of non-invasive tests for viability and ischaemia; comorbidities which may affect the safety of PCI (especially chronic kidney disease, peripheral vascular disease and conditions which might limit the safe use of DES). J-CTO score, Syntax score and Euroscore II should be calculated and recorded.

Several anatomical factors have been identified which predict CTO PCI success in contemporary practice and these have been incorporated into the J-CTO score. ${ }^{32}$ Low J-CTO scores are highly predictive of rapid antegrade wire crossing and ultimate case success and therefore may assist in determining the setting in which PCI is to be performed.

According to local practice, cases with J-CTO score of 0 in whom rapid antegrade wiring is considered likely may be reasonably treated by either 'non-CTO operators' or indeed by CTO teams.

Since success is not assured and complications are higher with more complex techniques, all but the simplest (J-CTO score 0) cases should be performed by dedicated CTO operators in centres with appropriate facilities. ${ }^{1}$ Dedicated CTO operators should have the full skill set to escalate from simple antegrade wiring to retrograde and dissection/re-entry at the same sitting. Dual arterial access is strongly encouraged unless no contralateral filling of the CTO vessel is seen.

A full range of equipment-including a range of CTO wires, short guide catheters for retrograde access, microcatheters, small over-the-wire balloons, snares, including intravascular ultrasound (IVUS) and catheter extension devices-should be available and the operator should be familiar with it. Emergency bailout kit should be immediately available in the catheter lab-echocardiography, pericardiocentesis and microvascular coils for perforations.

Adequate time should be set aside in the lab schedule to perform CTO PCI, which may be lengthy. Dual operator cases may be advantageous, especially with the most complex lesions including reattempts. Operators should liaise with local radiation protection advisors in order to set dose reference levels for CTOs. Close attention should be paid to radiation protection measures for operators-eye shields, table skirts, eyewear and disposable protection drapes should be available. Angiographic views should be altered during long cases and repeat procedures should be staged to reduce the risk of localised skin damage to patients. Ensuring patient safety is paramount and complex CTO procedures should always be performed in centres where colleagues with appropriate CTO experience are available to assist. Operators should carefully consider whether a complex CTO case would be most safely performed in a site with on-site cardiac surgery.

Restenosis is more common in CTO cases, at least partly related to the common requirement for long segment coverage. Drug-eluting stent use should therefore be the routine so an assessment of likely tolerability of prolonged dual antiplatelet therapy (DAPT) is required. Potential problems with DAPT should be highlighted and discussed at MDT.

\section{Key points: Ad hoc CTO PCI should not be performed}

- All CTOs should be discussed at MDT regardless of initial intended therapy.

- Complexity scoring of the CTO is recommended for all cases.

- All complex cases should be discussed with and/or performed by dedicated CTO operators.

- Drug-eluting stent use is strongly encouraged. 